\title{
To screen, identify and treat - how to improve nutritional care through the Malnutrition Universal Screening Tool (MUST)
}

\author{
E. Y. L. Leung ${ }^{1}$, S. T. White ${ }^{2}$, C. Richards ${ }^{1}$, E. Forrest ${ }^{2}$ and R. McKee ${ }^{1}$ \\ ${ }^{1}$ University Department of Surgery and ${ }^{2}$ Department of Gastroenterology, Glasgow Royal Infirmary, G4 OSF, UK
}

Undernutrition is often unrecognised and untreated ${ }^{(1)}$. The NHS Quality Improvement Scotland (QIS) has therefore made nutritional screening a mandatory standard for all hospital inpatients ${ }^{(2)}$. However, a regional audit in Lanarkshire ${ }^{(3)}$ has reported time limitations and the provision of adequate training were major obstacles. The aim of this audit was to review the implementation of nutritional screening, prevalence of undernutrition and the relationships between undernutrition and clinical parameters in patients admitted to our medical and surgical gastroenterological wards.

In total, the data of 203 (69 medical; 134 surgical) patients were prospectively collected between December 2009 and June 2010 . The MUST was the compulsory nutritional screening tool within the local health-care trust. Statistical analysis was performed using SPSS 17.0 software; non-parametric tests were used to compare between different groups when appropriate. The majority of our patients were $>65$ years $(56 \%)$, female $(54 \%)$, admitted as emergencies (57\%) and had moderate to high risk of undernutrition (MUST score $\geq 1 ; 53 \%)$. The risk of undernutrition did not appear to be associated with being under the care of physicians or surgeons $(P=0.216)$, gender $(P=0.538)$, emergency admission $(P=0.316)$ or age $(P=0.841)$. In contrast to previous reports ${ }^{(1)}$, younger $(\leq 50$ years $)$ patients appear to have similar risks of undernutrition compared to elderly ( $\geq 65$ years) patients ( $61 \% v .48 \%$ respectively). The implementation of nutritional screening is summarised below:

\begin{tabular}{lccc}
\hline & QIS standard (\%) & $\begin{array}{c}\text { Lanarkshire } \\
N 60(\%)\end{array}$ & $\begin{array}{c}\text { Local (Current) } \\
N 203(\%)\end{array}$ \\
\hline BMI recorded & 100 & 55 & 84 \\
Screening during admission & 100 & 50 & 78 \\
Food chart $^{\mathrm{a}}$ & 100 & 24 & 19 \\
Dietetic advice $^{\mathrm{b}}$ & 100 & 48 & 60 \\
Reassessment $^{\mathrm{c}}$ & 100 & 79 & 28 \\
\hline
\end{tabular}

${ }^{a}$ For MUST score $\geq 1$ only $(N 104)$. ${ }^{b}$ For MUST score $\geq 2$ only $\left(N\right.$ 80). ${ }^{c}$ For inpatient stay $\geq 7 \mathrm{~d}(N 145)$.

The median length of hospital stay (LOS) for all patients was 11 (7-19) d. Only age was significantly associated with overall LOS $(P=0.012)$. The risk of undernutrition appeared to be associated with the LOS in surgical patients $(P=0.032)$ with median LOS of MUST $=0$ and MUST $\geq 1$ being 11 (8-18) d and 13 (9-23) d, respectively. However, significant confounders exist (e.g. type of operations); it is unclear whether undernutrition is independently associated with LOS.

The present audit highlights the high prevalence $(>50 \%)$ of undernutrition among patients admitted to the gastroenterological wards, irrespective of age. The current management of high-risk patients is suboptimal and the reassessment rates were poor. The uptake rate of the MUST training has recently been improved through communication between nursing and dietetic hierarchies. Planned future audits will determine whether the changes made translate into clinical improvements.

Median (inter-quartile range).

1. Elia M (2003) The 'MUST' Report. BAPEN.

2. NHS Quality improvement Scotland (2003) Food, Fluids and Nutritional Care in Hospital.

3. NHS Lanarkshire (2008) A Critical Review of Training Needs on Providing Nutritional Care. 